\title{
Entropy Generation Minimization of Pin Fin Heat Sinks by Means of Metaheuristic Methods
}

\author{
Amir Jafary Moghaddam ${ }^{1 *}$ and Syfollah Saedodin ${ }^{1}$ \\ 'Department of Mechanical Engineering, Semnan Branch, Islamic Azad University, Semnan, Iran; \\ amirjafary145@yahoo.com; S_Sadodin@iust.ac.ir
}

\begin{abstract}
In this paper utilization of metaheuristic methods such as genetic algorithm and particle swarm optimization for determining design parameters of pin fin type heat sinks through minimizing generated entropy is discussed. This paper follows recent studies on entropy generation minimization of plate type heat sinks by evolutionary optimization methods.
\end{abstract}

Keywords: Pin Fin Heat Sink, Entropy Generation Minimization, Metaheuristic Algorithms.

\section{Introduction}

Heat sinks as cooling devices are used for heat transfer especially in electronic circuits. There are different structures and geometries of heat sinks which have different advantages of firmness, compactness and heat transfer quality. Most studied and designed heat sinks for electronic devices have been of plate fin geometry [4]. A plate fin heat sink is shown in Figure 1. To achieve good performance of plate fin heat sinks, entropy generation minimization is used in the paper of Culham and Muzychka [4], which introduces simultaneous optimization of geometric parameters, heat dissipation, used material and fluid flow conditions. Utilization of computational fluid dynamics for plate fin heat sink design is presented in [11]. A simple practical model for designing the plate fin heat sink as also proposed [16]. In recent years metaheuristic methods have been increasingly used for the problem of heat sink optimum design. Utilization of genetic algorithms [13], particle swarm optimization [17], imperialist competitive algorithm [18], and harmony search [19] for example have been proposed to find optimum values of parameters for plate fin heat sinks. Another geometry for heat sinks is pin fin geometry, for which pins of different cross section could be assumed (Figure 2). In Yu et al. [20] experimental study for comparison of fully plate fin heat sink and hybrid platepin fin heat sink is presented. Selection of pins cross section by CFD simulations and optimization has been proposed [12]. Entropy generation minimization for pin fin heat sinks is proposed in [8], and genetic algorithm based optimization of them is also presented in Hajabdollahi et al. [6] by means of thermal modeling.

In this paper different methods of metaheuristic optimization are used for minimization of generated entropy in heat transfer through pin fin heat sink by determining optimum parameters of its geometry. Previous studies have been done for utilization of some of these methods for plate fin heat sinks. The aim of this paper is to use two methods for pin fin heat sinks also, and then comparing the results. Using metaheuristic methods like GA

${ }^{*}$ Corresponding author:

Amir Jafary Moghaddam (amirjafary145@yahoo.com) 
and PSO is a general way to overcome hard optimization problems. In contrast to other case specific methods, these methods are based on simple principles and could be adapted to many problems with highest generalization. In section 2 of this paper, the concept of entropy generation minimization is introduced and modeling of pin fin heat sink entropy generation rate will be presented. In section 3 genetic algorithms are discussed. PSO and its modifications is the subject of section 4 and imperialist competitive algorithm is presented in section 5 . In section 6

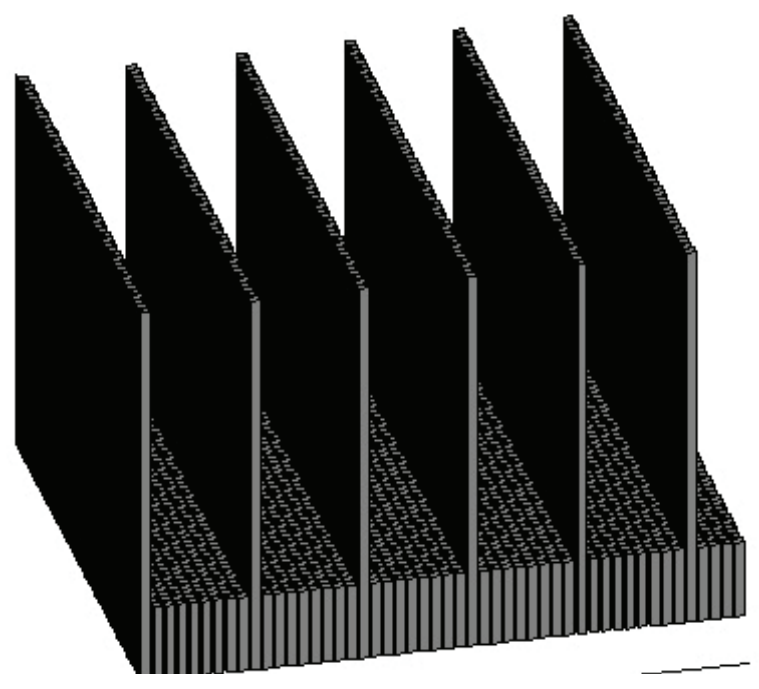

Figure 1. Heat sink with plate fin geometry.

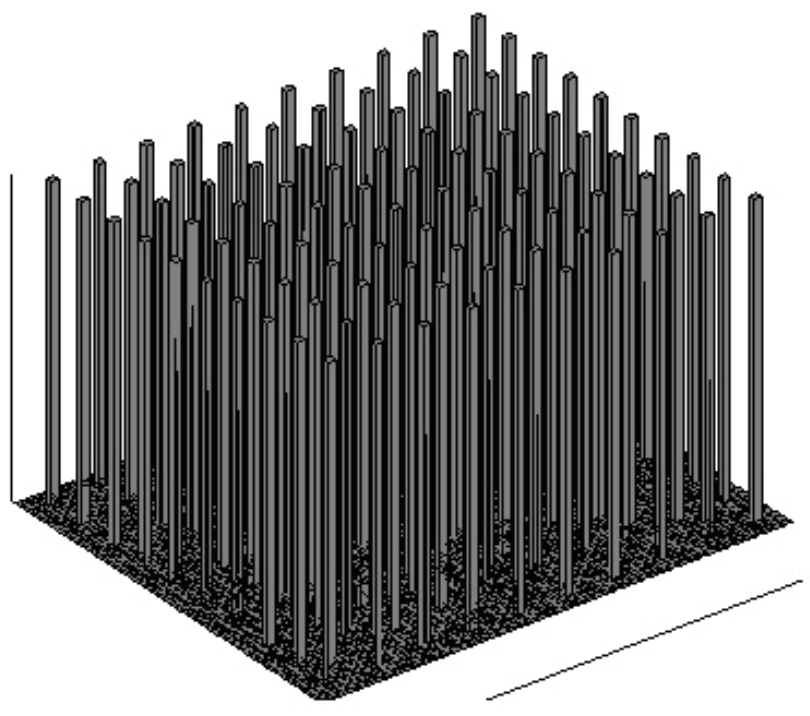

Figure 2. Heat sink with pin fin geometry. the mentioned metaheuristic methods are used for determining optimum parameters of pin fin heat sink through entropy generation minimization. Finally the results are discussed and compared.

\section{Entropy Generation Minimization}

Method of entropy generation minimization is introduced and developed by Bejan [2], to combine concepts of fluid dynamics and heat transfer for systems and models in which both aspects are present. This method is useful for finite-size and finite-time systems which are not considered as ideal infinite thermodynamic systems. The method is applicable in different domains of engineering and science such as low-temperature devices, heat transfer in electronic devices, and power plants.

The concept is based on assumption of a systemenvironment configuration depicted in Figure 3. In this configuration, the system has mass of $\mathrm{M}$, energy of $\mathrm{E}$ and entropy of $S$ at the moment, has an input mass transfer rate $\dot{m}_{i n}$ and an output mass transfer rate $\dot{m}_{\text {out }}$, and has heat transfer with $\mathrm{n}+1$ temperature reservoirs with heat transfer rates $\left(Q_{0}, Q_{1}, \ldots, Q_{n}\right)$. Also the net work done on system is $W$.

The first and second laws of thermodynamics for this system are in the forms of equation 1 and 2 respectively

$$
\begin{gathered}
\frac{d E}{d t}=\sum_{i=0}^{n} \dot{Q}_{i}-\dot{W}+\sum_{\text {in }} \dot{m} h^{0}-\sum_{\text {out }} \dot{m} h^{0} \\
\dot{S}_{\text {gen }}=\frac{d S}{d t}-\sum_{i=0}^{n} \frac{\dot{Q}_{i}}{T_{i}}-\sum_{\text {in }} \dot{m} s-\sum_{\text {out }} \dot{m} s
\end{gathered}
$$

In which $h^{0}$ is the sum of enthalpy and energy of boundary stream.

The method of entropy generation minimization will be the process of making the equation 2 to be its minimum possible value. Eliminating $\dot{Q}_{0}$ from equations 1 and 2 , and letting $\dot{S}_{g}=0$, one obtains the exergy analysis relation

$$
\begin{aligned}
& \dot{W}_{\text {rev }}=-\frac{d}{d t}\left(E-T_{0} S\right)+\sum_{i=1}^{n}\left(1-\frac{T_{0}}{T_{i}}\right) \dot{Q}_{i}+\sum_{\text {in }} \dot{m}\left(h^{0}-T_{0} S\right) \\
& -\sum_{\text {out }} \dot{m}\left(h^{0}-T_{0} S\right)
\end{aligned}
$$

We have also the relation

$$
\dot{W}_{r e v}-\dot{W}=T_{0} \dot{S}_{g e n}
$$




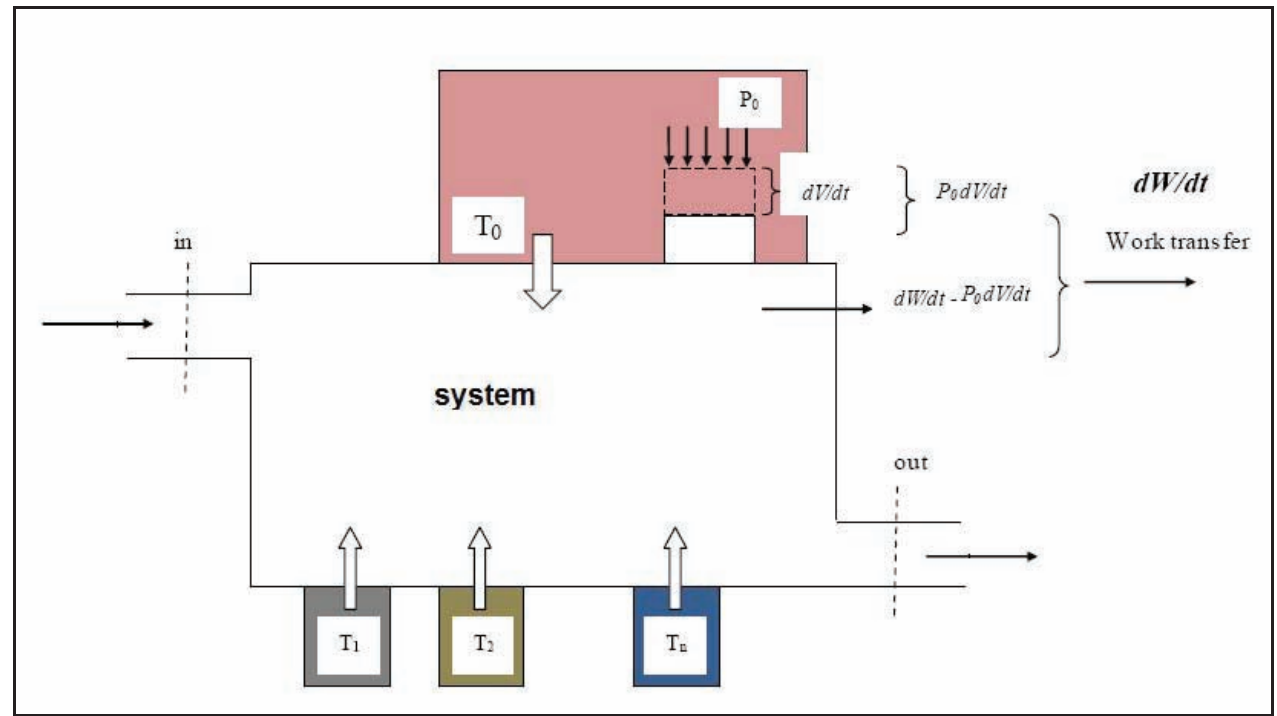

Figure 3. The system-environment configuration assumed for definition of entropy generation minimization method [2].

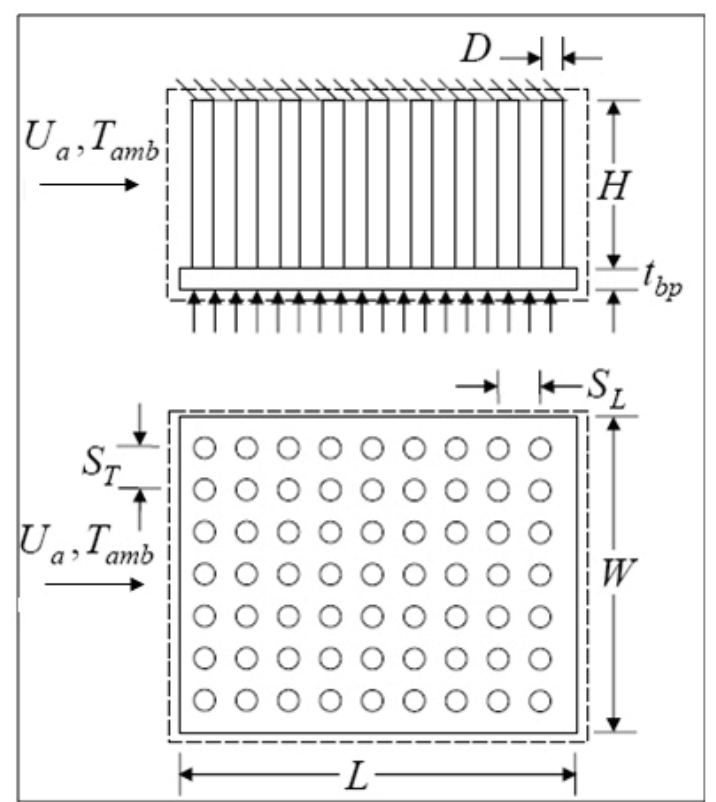

Figure 4. The schematic structure of pin fin heat sink.

which tells us the relation of destroyed power and entropy generation rate. So it is necessary to minimize entropy generation rate to gain best working performance of systems.

To design pin fin heat sink, in Khan et al. [8] the modeling of system for the aim of entropy generation minimization is developed. In this paper this modeling procedure is followed. The assumptions of this model are: Isotropic material, incompressible and uniform fluid, two-dimensional steady laminar flow with uniform velocity normal to pins axis and without bypass, and adiabatic fin tips. For the heat sink depicted schematically in Figure 4, one obtains the entropy generation rate as

$$
\dot{S}_{g e n}=\left(\frac{Q}{T_{a m b}}\right)^{2} R_{h S}+\frac{\dot{m} \Delta P}{\rho T_{a m b}}
$$

In equation 5, the resistance of heat sink is

$$
R_{h s}=R_{m}+R_{\text {fins }}
$$

in which the bulk material resistance is

$$
R_{m}=\frac{t_{b p}}{k A}
$$

and resistance of fins is

$$
R_{\text {fins }}=\frac{1}{\frac{N}{R_{c}+R_{\text {fin }}}+\frac{1}{R_{b p}}}
$$

for which we have

$$
\begin{aligned}
& R_{c}=\frac{1}{h_{C} A_{C}} \\
& R_{f i n}=\frac{1}{h_{f i n} A_{f i n} \eta_{f i n}} \\
& R_{b p}=\frac{1}{h_{b p} A_{b p}}
\end{aligned}
$$


defined using

$$
\begin{aligned}
& \eta_{\text {fin }}=\frac{\tanh (m H)}{m H} \\
& m=\sqrt{\frac{4 h_{\text {fin }}}{k D}}
\end{aligned}
$$

For an inline array of cylindrical fins a dimensionless heat transfer coefficient is defined as

$$
N u_{D f i n}=\frac{h_{f i n} D}{v}=C_{1} \operatorname{Re}_{D}^{\frac{1}{2}} \operatorname{Pr}^{\frac{1}{3}}
$$

and $C_{1}$ is determined by geometric parameters as

$$
C_{1}=\frac{\left[0.2+\exp \left(-0.55 S_{T}\right)\right] S_{T}^{0.775} S_{L}^{0.212}}{\left(S_{T}-1\right)^{0.5}}
$$

For base plate of heat sink, heat transfer coefficient is also shown that to be

$$
N u_{L}=\frac{h_{b p} L}{v}=0.75 \operatorname{Re}_{L}^{\frac{1}{2}} \operatorname{Pr}^{\frac{1}{3}}
$$

Mass flow rate and pressure difference are calculated by

$$
\begin{aligned}
& \dot{m}=\rho U_{a} N_{T} S_{T} H D \\
& \Delta P=f\left(\rho U_{\text {max }^{2}}\right) / 2 N_{L}
\end{aligned}
$$

The pressure drop is also related to structural parameters as

$$
f=K_{1}\left\{0.233+\frac{45.78}{\left(S_{T}-1\right)^{1.1} R e_{D}}\right\}
$$

in which

$$
K_{1}=1.009\left(\frac{S_{T}-1}{S_{L}-1}\right)^{\frac{1.09}{R e_{D}^{0.0533}}}
$$

The maximum flow velocity is

$$
U_{\max }=\max \left\{\frac{S_{T}}{S_{T}-1} U_{a}, \frac{S_{T}}{S_{D}-1} U_{a}\right\}
$$

with definition of

$$
S_{D}=\sqrt{S_{L}^{2}+\left(\frac{S_{T}}{2}\right)^{2}}
$$

The resulting optimization problem is a nonlinear one, in which the optimum values of heat sink parameters are to be determined subject to constraints on assumed values for some of system temperatures and sizes. So it is useful to utilize metaheuristic methods to solve this nonlinear problem. In the next sections some of such methods are discussed.

\section{Genetic Algorithm (GA)}

One of the most well-known metaheuristic optimization methods is genetic algorithm which is based on a model of evolution of chromosomes [5]. GA is used in many applications of engineering in recent years [1]. This algorithm selects the most fitting solutions of a problem due to a predefined fitness function. As in evolutionary theory the best individuals in a generation are the most probable to survive and reproduce. In GA, next generation of solutions (chromosomes) are determined by means of some basic operations on chromosomes of current generation. The crossover operation makes new individuals by combining some parts of a solution with some parts from another solution, and it may be considered as a model for reproduction. Another operation is the mutation in which values of some elements of some chromosomes will change randomly to another values; as a model for mutations of genes in nature. It is also possible for some of the most fitting chromosomes to survive and be present in next generation directly. The main procedure of GA is as follows:

1. An initial set of chromosomes (possible solutions of the problem) are set as initial population. Each chromosome is a vector of numbers (genes), and each of this numbers defines one of optimization variables.

2. For all individuals in population, the fitness is calculated by fitness function of problem. Then individuals are sorted due to their fitness values.

3. Probabilities of crossover, mutation and direct transfer are determined from fitness values for each individual.

4. In crossover process, some pairs of individuals are selected (with a selection strategy) and make new individuals (offsprings) as shown in Figure 5. 


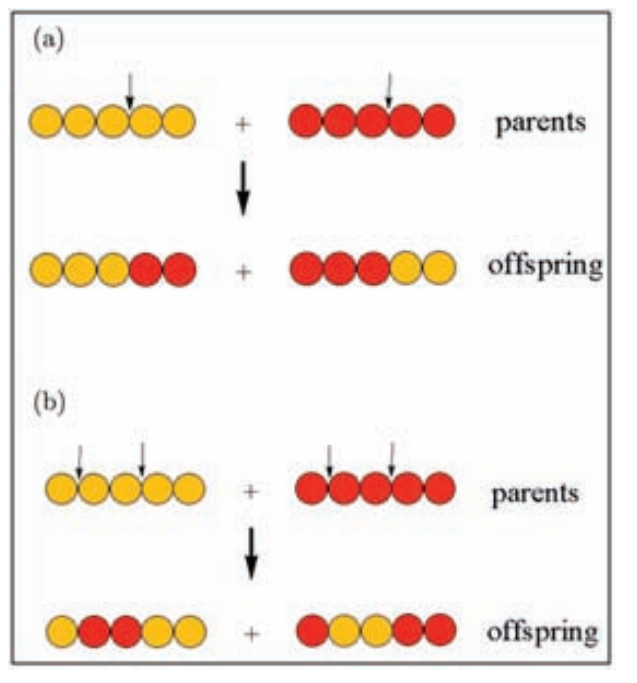

Figure 5. Crossover operation in GA.

5. In mutation process, some individuals are selected and some of their elements are changed randomly.

6. Some of most fitting individuals are selected to go to the next generation directly and with no change.

7. New generation is made from crossover, mutation and direct transfer. Then the procedure from stage 2 is iterated for new generation.

By the iterative procedure mentioned above, the new generations will get better gradually. After some number of iterations the population converges to optimum values. The flowchart for main operations in GA is shown in Figure 6.

\section{Particle Swarm Optimization (PSO)}

Particle Swarm Optimization is another well-known metaheuristic optimization method which is introduced in Kennedy and Eberhart [7] by modeling the movements of a swarm in a search space. The possible solutions of the problem are set to be the possible positions of particles in the search space, and the fitness function determines the fitness of each position. The particles have information sharing about their best experienced position. There is a memory for swarm to maintain the best experienced position from all particles in the swarm. Also, each particle has its own memory for its best experienced position. The velocity of a particle is determined from the sum of vectors from particle position pointed to best positions (Figure 7).

The new position of particle will be determined from this velocity and the procedure of updating the particle's

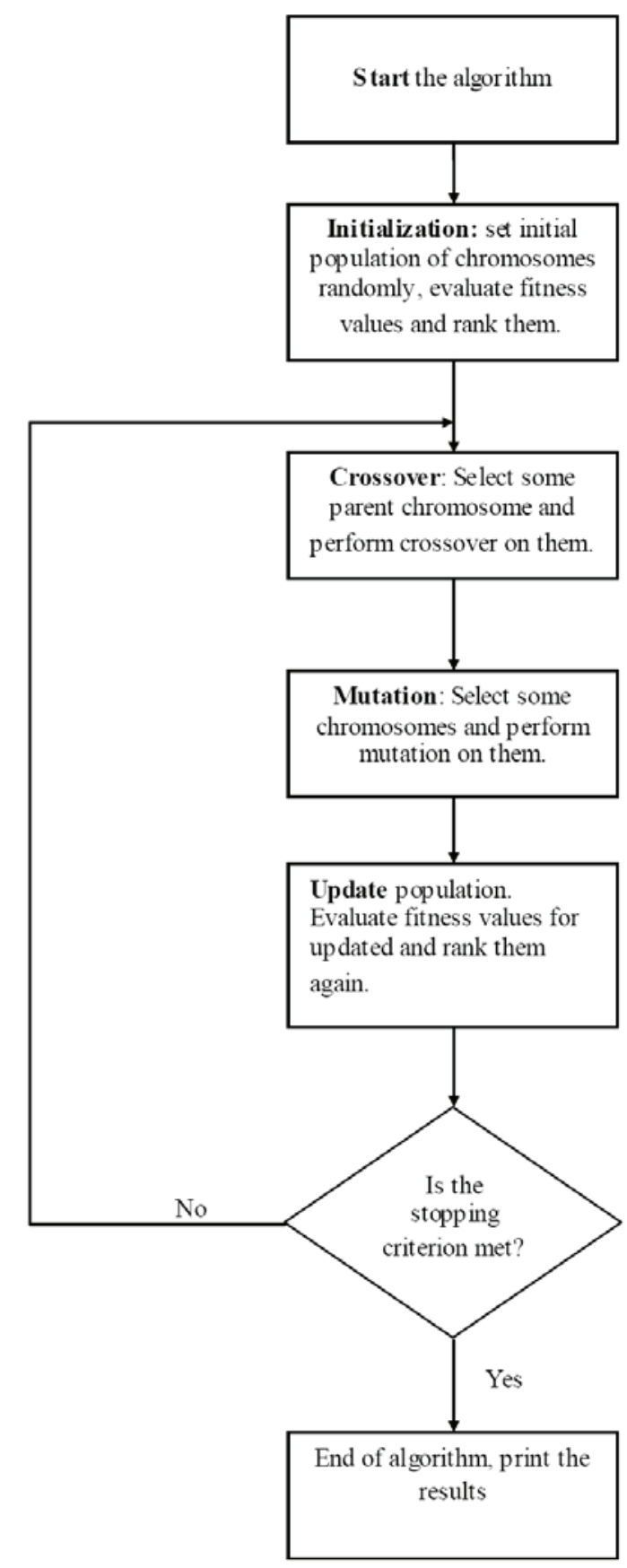

Figure 6. Flowchart of GA.

velocity and position is iterated. The main procedure of PSO is as follows:

1. Initial positions and velocities of particles in search space are set randomly. For each particle the fitness of its position is determined by fitness function. The best fitting position among all swarm is set as global best position (gbest), and for $i$-th particle, its position is set for personal best position ( pbest $_{i}$ ). 


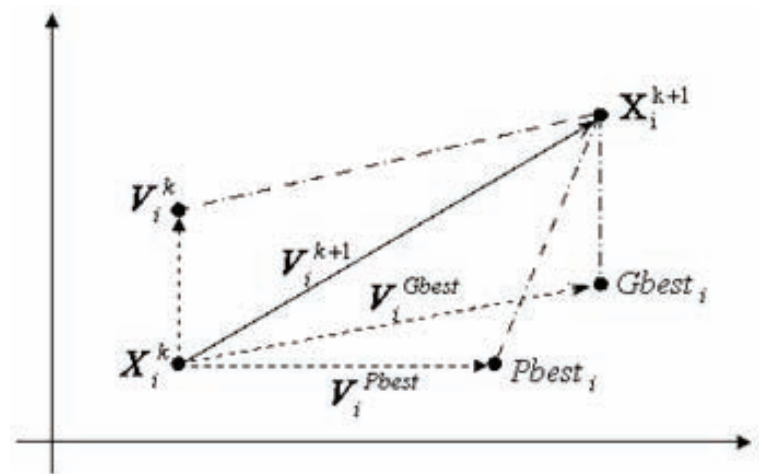

Figure 7. Determining the velocity of particle in PSO.

2. Velocity of $i$-th particle is updated as equation (19)

$$
\begin{aligned}
& V_{i}(t+1)=I V_{i}(t)+r_{1} C_{1}\left(\text { gbest }-x_{i}(t)\right) \\
& +r_{2} C_{2}\left(\text { pbest }_{i}-x_{i}(t)\right),
\end{aligned}
$$

where $I$ is inertia factor, $r_{1}$ and $r_{2}$ are random numbers, and $C_{1}$ and $C_{2}$ are constant coefficients.

3. Position of $i$-th particle is updated also, as equation (20)

$$
x_{i}(t+1)=x_{i}(t)+\delta V_{i}(t+1),
$$

in which the $\delta$ determines the time step.

4. Fitness values for the new positions are calculated by fitness function, gbest and pbest values are updated, and the procedure is iterated from stage 2 .

After enough numbers of iterations, the swarm converges to the position with optimum fitness. This best position represents the optimum values for optimization variables. The flowchart for conventional PSO algorithm is depicted in Figure 8.

Some modifications of PSO are proposed in literature. In Multi-Population PSO (MPSO), the whole swarm is divided to some subpopulations, which are evolved separately or have some sorts of information sharing and migrations of particles from subpopulations to another ones [10]. In Fuzzy PSO (FPSO), main equations of PSO are considered to have fuzzy variables [14]. Chaotic and Quantum PSO (CPSO and QPSO) are modifications of PSO with local search properties $[15,9]$. PSO is used in many applications of engineering in recent years [3].

\section{Results and Discussion}

In this section the results of entropy generation minimization of pin fin heat sink by GA and PSO algorithms are presented. The main task is to find design parameters $D, H$,

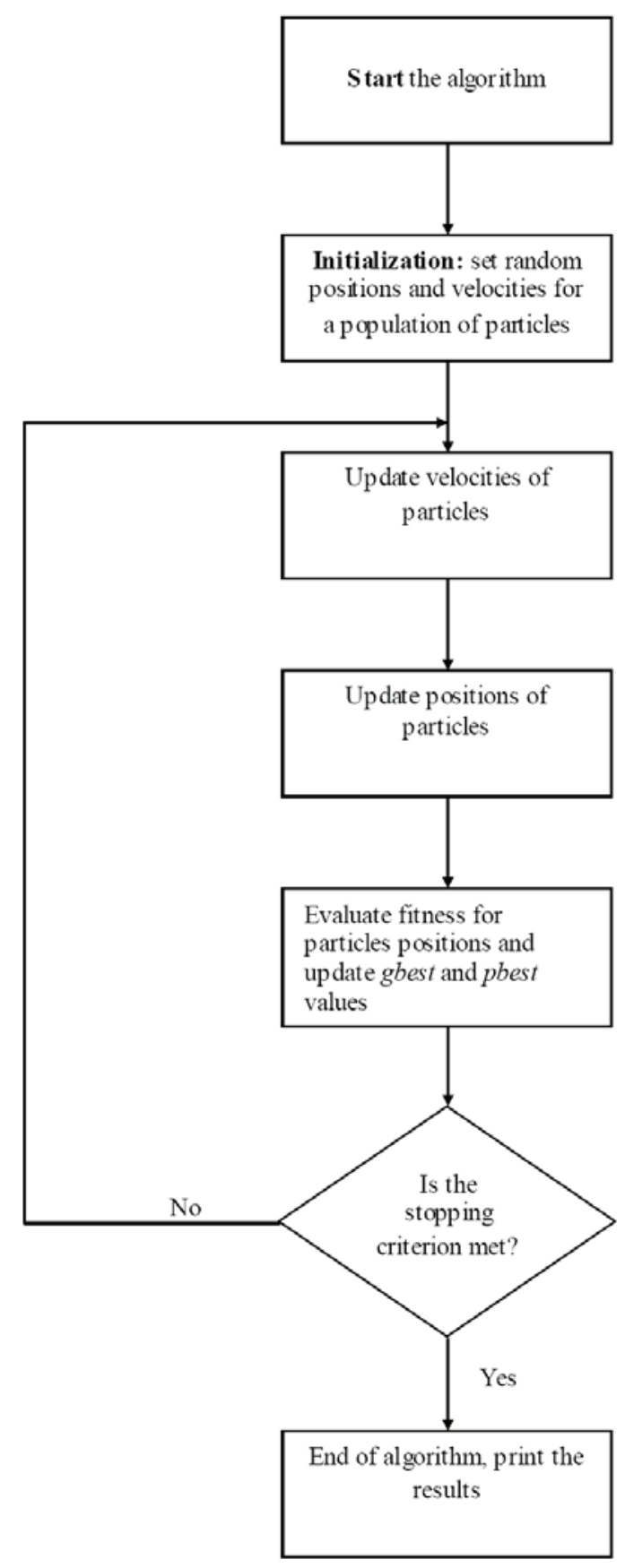

Figure 8. Flowchart of PSO.

$U_{a}, N_{T}$ and $N_{L}$ so the entropy generation rate of equation (5) be in its minimum value.

For GA the chromosome is set to be a vector of numbers each presenting one of the mentioned design variables. For PSO the design variables are coordinates values of particle positions. For sake of comparison both population sizes in GA and PSO are set with same value and equal to 25, and maximum iterations of algorithms are set to 165 . 
The convergence curves of GA and PSO, -which show the minimum entropy generation rate in each iteration- are shown in Figures 9 and 10 respectively. Both GA and PSO have found optimum solutions for design parameters finally, but PSO have faster behavior, both in computational time and in finding the optimal solution. The results are summarized in Table 1.

\section{Conclusion}

In this paper metaheuristic methods of GA and PSO are utilized for designing pin fin heat sink with objective of minimization of generated entropy. Both methods could find optimum solutions for design variables properly, but PSO was faster in finding the solution and in total elapsed computation time. Results from this study showed that

Table 1. Results of entropy generation minimization with GA and PSO

\begin{tabular}{lll}
\hline & \multicolumn{1}{c}{ GA } & \multicolumn{1}{c}{ PSO } \\
\hline $\begin{array}{l}\text { Optimum design } \\
\left(D, H, U_{a}, N_{T}, N_{L}\right)\end{array}$ & $(4 \mathrm{~mm}, 12 \mathrm{~mm}, 10,10)$ & $(4 \mathrm{~mm}, 12 \mathrm{~mm}$, \\
$\begin{array}{l}\text { Minimum Entropy } \\
\text { Generation rate }\end{array}$ & $0.00148 \mathrm{~W} / \mathrm{K}$ & $0.00148 \mathrm{~W} / \mathrm{K}$ \\
$\begin{array}{l}\text { Total Elapsed } \\
\text { computation time }\end{array}$ & $15.08 \mathrm{sec}$ & $5.73 \mathrm{sec}$ \\
$\begin{array}{l}\text { Iteration of finding } \\
\text { the optimum }\end{array}$ & 35 & 5 \\
\hline
\end{tabular}

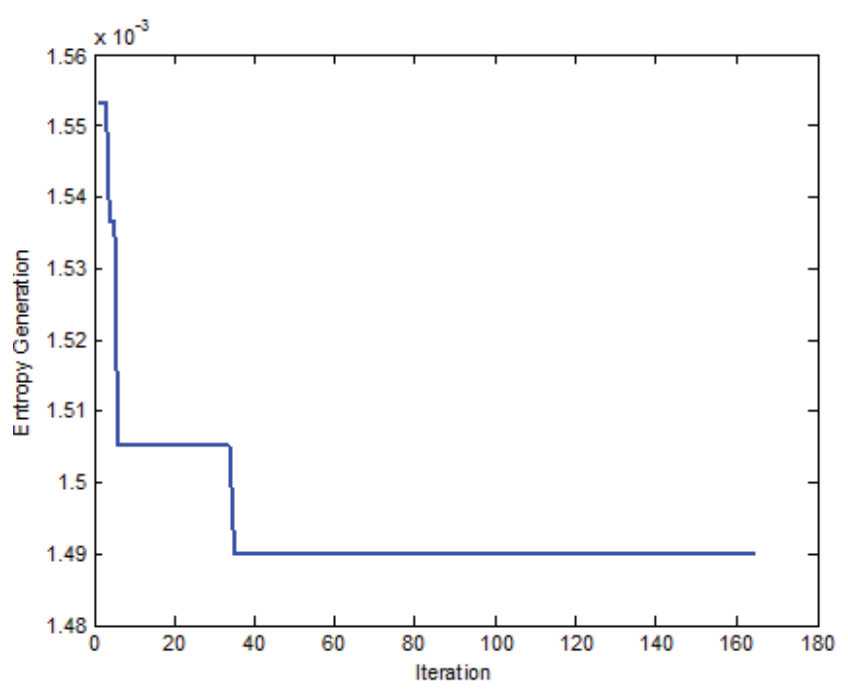

Figure 9. Convergence curve for GA.

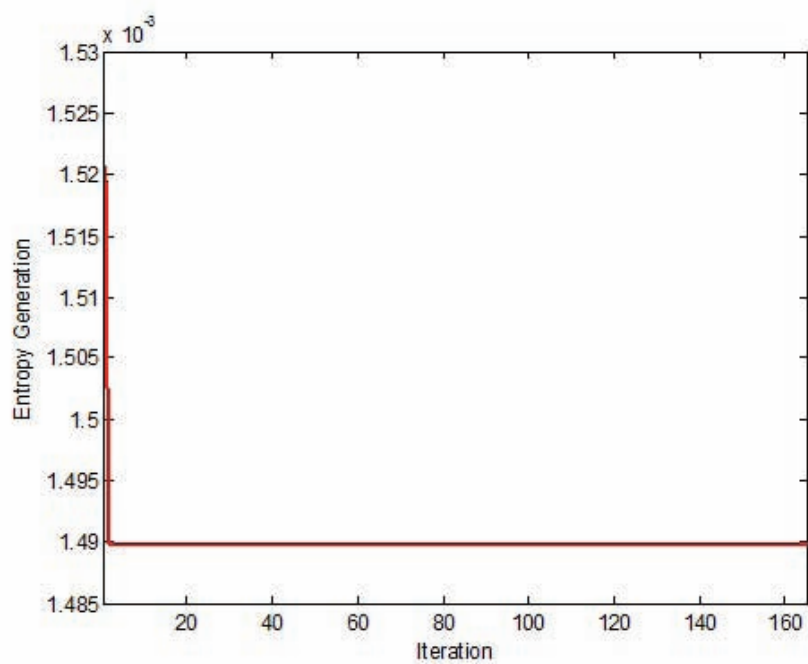

Figure 10. Convergence curve for PSO.

using metaheuristic methods one can find more suitable designs for pin fin geometry of heat sinks which leads to more efficiency of electronic devices, and lower risks of failures and damages due to generated heat.

\section{References}

1. Aref, A., Davoudi, M., \& Davoudi, M. (2012). Optimal placement and estimation of DG capacity in distribution network's using Genetic Algorithm-based method. Indian Journal of Science and Technology, 5(3), 2258-2264.

2. Bejan, A. (1996). Entropy generation minimization: The new thermodynamics of finite-size devices and finite-time processes. Journal of Applied Physics, 79(3), 1191-1218.

3. Boroujeni, S. M. S., Hemmati, R., Delafkar, H., \& Boroujeni, A. S. (2011). Optimal PID power system stabilizer tuning based on particle swarm optimization. Indian J. Sci. Technol, 4(4), 379-383.

4. Culham, J. R., \& Muzychka, Y. S. (2001). Optimization of plate fin heat sinks using entropy generation minimization. Components and Packaging Technologies, IEEE Transactions on, 24(2), 159-165.

5. Goldberg, D. E. (1989). Genetic algorithms in search, optimization, and machine learning.

6. Hajabdollahi, F., Rafsanjani, H. H., Hajabdollahi, Z., \& Hamidi, Y. (2012). Multi-objective optimization of pin fin to determine the optimal fin geometry using genetic algorithm. Applied Mathematical Modelling, 36(1), 244-254.

7. Kennedy, J., \& Eberhart, R. (1995, November). Particle swarm optimization. In Neural Networks, 1995. Proceedings., IEEE International Conference on (Vol. 4, pp. 1942-1948). IEEE.

8. Khan, W. A., Culham, J. R., \& Yovanovich, M. M. (2005). Optimization of pin-fin heat sinks using entropy generation 
minimization. Components and Packaging Technologies, IEEE Transactions on, 28(2), 247-254.

9. Liu, B., Wang, L., Jin, Y. H., Tang, F., \& Huang, D. X. (2005). Improved particle swarm optimization combined with chaos. Chaos, Solitons \& Fractals, 25(5), 1261-1271.

10. Niu, B., Zhu, Y., \& He, X. (2005). Multi-population cooperative particle swarm optimization. Advances in Artificial Life, 874-883.

11. Park, K., Oh, P. K., \& Lim, H. J. (2006). The application of the CFD and Kriging method to an optimization of heat sink. International journal of heat and mass transfer, 49(19), 3439-3447.

12. Sahiti, N., Durst, F., \& Geremia, P. (2007). Selection and optimization of pin cross-sections for electronics cooling. Applied thermal engineering, 27(1), 111-119.

13. Sanaye, S., \& Hajabdollahi, H. (2010). Thermal-economic multi-objective optimization of plate fin heat exchanger using genetic algorithm. Applied Energy, 87(6), 1893-1902.

14. Shi, Y., \& Eberhart, R. C. (2001). Fuzzy adaptive particle swarm optimization. In Evolutionary Computation, 2001. Proceedings of the 2001 Congress on (Vol. 1, pp. 101-106). IEEE.
15. Sun, J., Feng, B., \& Xu, W. (2004, June). Particle swarm optimization with particles having quantum behavior. In Evolutionary Computation, 2004. CEC2004. Congress on (Vol. 1, pp. 325-331). IEEE.

16. Wu, H. H., Hsiao, Y. Y., Huang, H. S., Tang, P. H., \& Chen, S. L. (2011). A practical plate-fin heat sink model. Applied Thermal Engineering, 31(5), 984-992.

17. Yousefi, M., Enayatifar, R., \& Darus, A. N. (2011a). Optimal design of plate-fin heat exchangers by a hybrid evolutionary algorithm. International Communications in Heat and Mass Transfer.

18. Yousefi, M., Darus, A. N., \& Mohammadi, H. (2011b). Second law based optimization of a plate fin heat exchanger using Imperialist Competitive Algorithm.

19. Yousefi, M., Enayatifar, R., Darus, A. N., \& Abdullah, A. H. (2012). Optimization of Plate-fin heat exchangers by an improved harmony search algorithm. Applied Thermal Engineering.

20. Yu, X., Feng, J., Feng, Q., \& Wang, Q. (2005). Development of a plate-pin fin heat sink and its performance comparisons with a plate fin heat sink. Applied thermal engineering, 25(2), 173-182. 